International Journal of Biomedicine I June 2019 - Volume 9, Issue Suppl_1: Abstracts From the Second Russian International Conference "Cryo-electron microscopy 2019: achievements and prospects"

ORAL ABSTRACT PRESENTATIONS

SESSION TITLE: Structure of membrane proteins

DOI: 10.21103/IJBM.9.Suppl_1.OR1

\title{
Abstract OR-1: NMR Spectroscopy: Alternative Method for Structural Studies of Highly Dynamic Membrane Proteins and Ion Channels
}

\author{
Mikhail Yu. Myshkin, Dmitrii S. Kulbatskii, Anton O. Chugunov, Atonina A. Berkut, Alexander S. \\ Paramonov, Alexander A. Vassilevski, Ekaterina N. Lyukmanova, Zakhar O. Shenkarev \\ Shemyakin-Ovchinnikov Institute of Bioorganic Chemistry, Moscow, Russia
}

Background: Integral membrane proteins (IMPs) play key roles in a variety of biological processes essential for living cells and multicellular organisms. Despite the intensive studies, the molecular mechanisms of IMPs functioning are not very well established. IMPs frequently possess highamplitude intramolecular mobility, which is essential for their function, but hampers structure-function studies. High-resolution NMR spectroscopy is complimentary to crystallographic and electron microscopy methods and permits simultaneous characterization of the structure and dynamics of moderately sized (up to $30 \mathrm{kDa}$ ) IMPs.

Methods: First and second isolated voltage-sensing domains (VSD, 150 a.a., four transmembrane helices, S1-S4) of human skeletal muscle sodium channel Nav1.4 were produced by cellfree expression. The structure, dynamics, and interaction of VSDs with gating modifier toxin Hm-3 (crab spider Heriaeus melloteei) were studied by heteronuclear $\left({ }^{1} \mathrm{H} /{ }^{13} \mathrm{C} /{ }^{15} \mathrm{~N}\right) \mathrm{NMR}$ spectroscopy.

Results: The VSDs samples demonstrated limited stability in lipid- and detergent-based membrane mimetics due to aggregation (half-life time $\sim 24 \mathrm{~h}$ ). Nevertheless, the use of combinatorial selective ${ }^{13} \mathrm{C},{ }^{15} \mathrm{~N}$-labeling permitted to assign $~ 50 \%$ of VSDs backbone. The secondary structure of VSDs showed similarity with the known structures of other $\mathrm{K}^{+}$and $\mathrm{Na}^{+}$channels. Paramagnetic relaxation enhancement and PELDOR EPR data obtained for the spin-labeled domains confirmed the native-like tertiary structure of isolated VSDs. ${ }^{15} \mathrm{~N}$ relaxation data revealed significant mobility of both VSDs at psns time scale. The instability of the domain structure associated with these motions is probably responsible for the observed protein aggregation. Hm-3 has similar affinities to both VSDs, but binds to the different sites on their surface. At VSD-I the toxin interacts with the S3-S4 extracellular loop forming salt bridges with conserved E208 and D211 residues, while at VSD-II the toxin binds to the S1-S2 extracellular loop interacting with E604 and D606 residues. In the both cases, the toxin binding to these charged groups probably inhibits voltage sensor transition to the activated state and blocks the channel opening. 

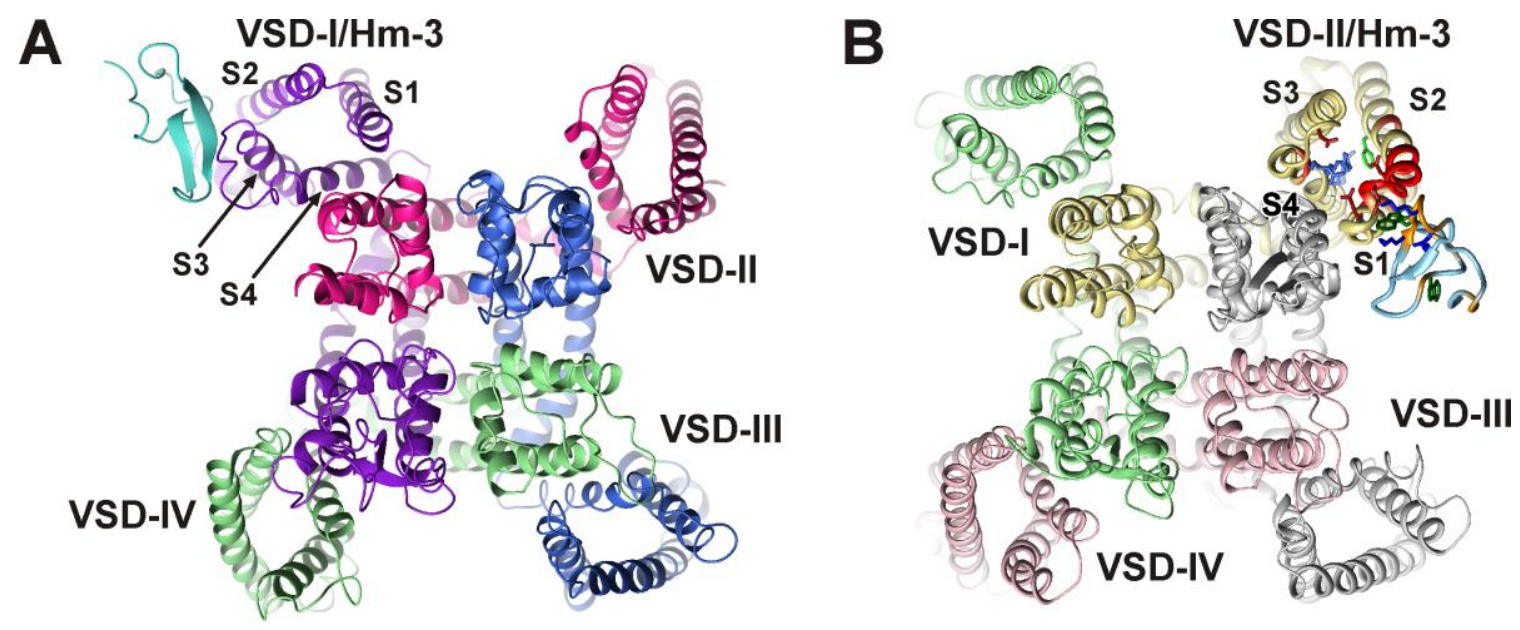

Fig. 1. Obtained models of Hm-3 complexes with Nav1.4 channel.

Conclusion: Obtained results validate the use of NMR spectroscopy for the ligand-receptor interactions in the conformationally flexible domains of ion channels.

Key Words: sodium channel $\bullet$ gating modifier $\bullet$ combinatorial selective labeling ligand-receptor interactions

Sources of Funding: This work was supported by the Russian Science Foundation (Project № 16-14-10338).

International Journal of Biomedicine. 2019;9 Suppl 1: S6. doi: 10.21103/IJBM.9.Suppl_1.OR1

C2019 International Medical Research and Development Corporation 